\title{
Preparation of Biodegradable Materials by Reactive Extrusion
}

\author{
I. Moura ${ }^{1, a}$, A.V. Machado ${ }^{1, b}$, F.M. Duarte ${ }^{1, c}$, G. Botelho ${ }^{2, d}$ and R. Nogueira ${ }^{3, e}$ \\ ${ }^{1}$ IPC - Instituto de Polímeros e Compósitos, University of Minho, 4800-058 Guimarães, Portugal \\ ${ }^{2}$ Centro de Química, University of Minho, Campus de Gualtar, 4710-057 Braga, Portugal
}

${ }^{3}$ Centro de Engenharia Biológica, University of Minho, Campus de Gualtar, 4710-057 Braga, Portugal

aisabelm@dep.uminho.pt, ${ }^{\mathrm{b}} \mathrm{avm} @$ dep.uminho.pt, ${ }^{\mathrm{c} f d u a r t e @ d e p . u m i n h o . p t,}$ gbotelho@quimica.uminho.pt, ${ }^{\mathrm{e}}$ regina@deb.uminho.pt

Keywords: Biodegradable materials, Blends, PCL, PLA.

\begin{abstract}
This work aimed to prepare biodegradable polymeric materials based on blends of a synthetic high density polyethylene (HDPE) and biodegradable polymers such as polylactic acid (PCL) and poly(caprolactone) (PLA), in a co-rotating twin-screw extruder. A polyethylene modified with maleic anhydride was used as compatibiliser. The mechanical results showed that the addition of PLA improves the blends stiffness while the addition of PCL leads to materials with a greater elongation at break and a lower Young modulus. This feature is related with the mechanical properties of each material as well as the adhesion between them. Concerning the biodegradability tests, it was found that HDPE/PCL blend presents the highest degree of biodegradability.
\end{abstract}

\section{Introduction}

During the last decades, the use of polymeric materials has grown progressively and now, these materials are one of the most attractive categories of materials. This success is mainly related to their low cost, good mechanical properties, aesthetic qualities and resistance to physical ageing and biological attacks. However, the degrading resistance of synthetic polymers, especially in such applications where they are used for a short period of time before becoming waste, the growing environmental awareness and the new environmental regulations are forcing the industries to seek for more ecologically friendly materials for their products. The most desirable long-term solution to this problem is the use of biodegradable plastics, which undergo controlled biodegradation through the action of living organisms, the most environmentally compatible pathway for degradation [1].

Most biodegradable polymers have good properties comparable to many petroleum-based plastics and readily biodegradable, having the potentiality to compete with commodity plastics. However, their relative poor physical properties, such as high brittleness, moisture sensitivity, low heat distortion temperature, difficult their processability, restricting their use in a wide-range of applications [2]. The long-term properties of renewable materials are also very important especially if the products are not single use applications. Therefore, new approaches to develop usable biodegradable materials are a subject of increasing research interest [1, 3]. For example, starch can be processed as thermoplastic polymers if plasticizer is added. Such behaviour is similar to that exhibited by cellulose macromolecules. Ring opening copolymerisation of lactides, $\varepsilon$-caprolactone and other monomers has been also studied and the properties observed were in a widely range from flexible elastomers to stiff plastics. Blends of both starch and aliphatic polyester such as poly- $\varepsilon-$ caprolactone were studied but the system exhibit poor interfacial adhesion. Preparation of blends with synthetic polymers [4-6] or conventional composites using inorganic or natural fillers [7], are among the routes to improve some of the properties of biodegradable polymers, modifying degradation rates and reducing the cost of the final materials.

The objective of this work is to prepare biodegradable polymeric materials based on blends of a synthetic and a biodegradable polymer. Thus, blends of high density polyethylene (HDPE), polylactic acid (PLA) and poly(c-caprolactone) (PCL) using polyethylene modified with maleic anhydride (PE-g-MA) as a compatibiliser were prepared in a co-rotating twin-screw extruder. 
Morphological and mechanical properties tests were performed. Biodegradability of the samples was evaluated using pure culture of Pseudomonas fluorescens.

\section{Experimental}

Materials. High density polyethylene (HDPE, 2008SN60) supplied by Total, polyethylene modified with maleic anhydride (PE-g-MA, Lotader 3210) supplied by Arkema, polycaprolactone (PCL) CAPA FB100 kindly supplied by Solvay and polylactic acid (PLA) NatureWorks ${ }^{\circledR}$ PLA Polymer 2002D from Novamont were used. The compositions of the samples used in this work are shown in Table 1.

Table 1 - Composition of the samples

\begin{tabular}{ccccccc}
\hline \multirow{2}{*}{ Sample } & HDPE & & PCL & & PLA & PE-g-MA \\
\cline { 2 - 2 } \cline { 6 - 7 } \cline { 5 - 6 } & {$[\mathrm{wt} \%]$} & & {$[\mathrm{wt} \%]$} & & {$[\mathrm{wt} \%]$} & {$[\mathrm{wt} \%]$} \\
\hline 1 & 100 & & 0 & & 0 & 0 \\
2 & 30 & & 0 & & 60 & 10 \\
3 & 30 & & 60 & & 0 & 10 \\
\hline
\end{tabular}

Compounding. The blend components were tumble mixed and processed in a Leistritz LSM 30.34 laboratory modular co-rotating twin screw extruder using a barrel set temperature of $190{ }^{\circ} \mathrm{C}$, a screw speed of $100 \mathrm{rpm}$ and a throughput of $3 \mathrm{~kg} \mathrm{~h}^{-1}$. The screw contains a series of transport elements separated by three mixing zones, consisting of staggered kneading disks and a left-hand element, respectively (fig. 1). HDPE and blended materials were injection moulded at the temperature of $190^{\circ} \mathrm{C}$ to obtain dumb-bell specimens, on which mechanical tests were performed.

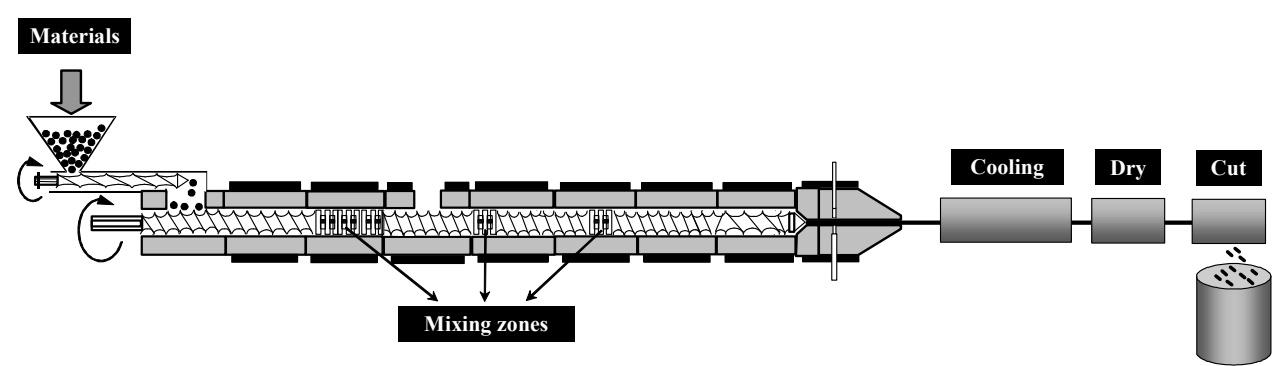

Figure 1 - Laboratory modular Leistritz LSM 30.34.

\section{Material characterization}

Mechanical Properties. The uniaxial tensile properties of the materials (tensile specimens) were determined using an Instron 4505 Tensile Machine at crosshead speed $50 \mathrm{~mm} \mathrm{~min}^{-1}$, relative humidity of $50 \%$ and temperature of $23{ }^{\circ} \mathrm{C}$. An average of 10 tests was performed to calculate the elongation at break and modulus values.

Morphology. HDPE/PLA and HDPE/PCL blends were fractured in liquid nitrogen and then gold plating, the morphology was analysed using a FEI Quanta 400 Scanning Electron Microscope.

Biodegradability. A pure culture of Pseudomonas fluorescens was used in static culture growing experiments carried out with HDPE and mixtures of HDPE+PLA and HDPE+PCL as sole carbon and energy sources. Each polymer (shaped as a disc with $25 \mathrm{~mm}$ diameters and thickness of 0.25 $\mathrm{mm}$ ) was placed into a sterilised bottle which was filled with R2A medium (prepared without organic carbon) and then spiked with the pure culture directly from an agar plate. Bacterial growth 
both attached to the surface of the polymer and in suspension was monitored by carrying out a series of total cell counts over a period of 7 weeks and cells were enumerated by epifluorescence microscopy after DAPI staining.

\section{Results and Discussion}

Fig. 2 presents the elongation at break and the Young modulus for all tested samples. As expected, the mechanical properties depend on the polymer that is blended with HDPE. The addition of PLA increases the rigidity of the material, yielding a material with a higher modulus and a lower elongation at break, while the addition of PCL increases the elongation at break and decreases the modulus. The different behaviour can be explained by the mechanical properties of the individual components (HDPE, PLA and PCL) and by the morphology of the HDPE/PLA and HDPE/PCL blends. As it is known, HDPE has good mechanical properties, both elongation at break and modulus. Conversely, PLA is a material with high rigidity, high modulus and low elongation at break while PCL has low modulus and high elongation at break.
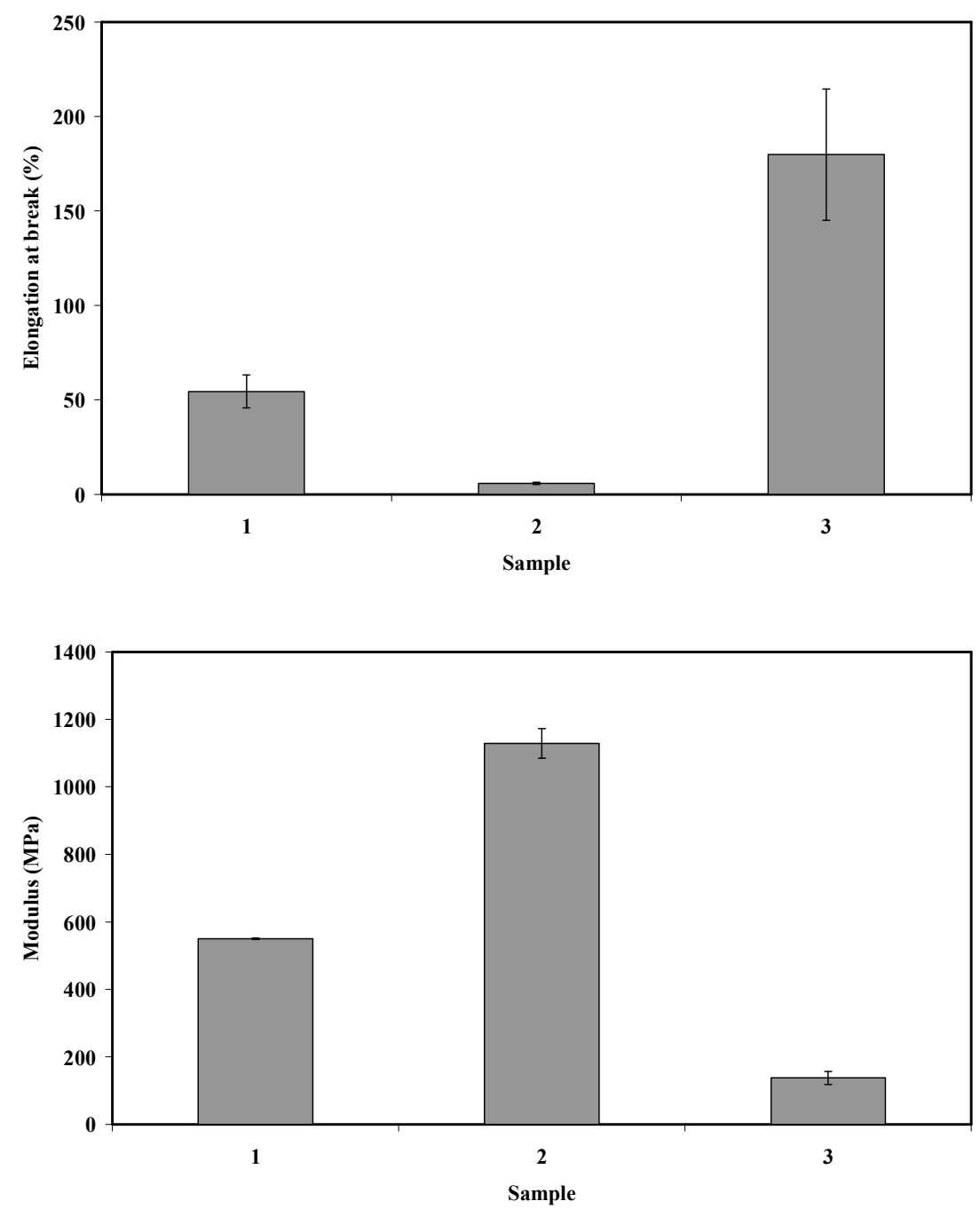

Figure 2 - Elongation at break and Young modulus for all samples.

The morphology of the HDPE/PLA and HDPE/PCL blends presented in fig. 3, also explains differences observed in mechanical behaviour, indicated above. In fig. $3 \mathrm{a}$ it is possible to see that the adhesion between PLA and HDPE is not good, elongated droplets of PE can be detected in the PLA matrix. Even with the addition of PE-g-MA, which acts as a compatibiliser increasing the 
interfacial adhesion due the chemical interaction between the hydroxyl group of PLA and anhydride group of PE-g-MA, the adhesion is not good enough to improve the elongation at break. In opposition, a good adhesion can be observed in the HDPE/PCL blend (fig. 3b) and in this case it is not easy to detect the polyethylene dispersed phase in the PCL matrix.

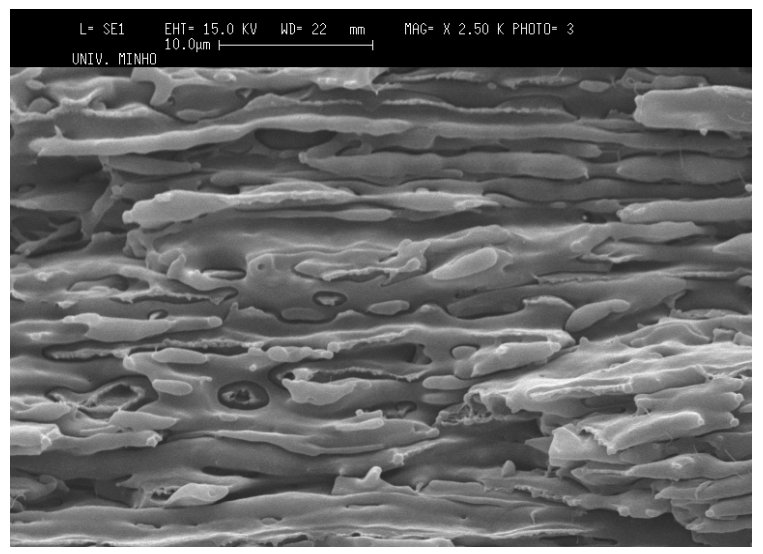

a)

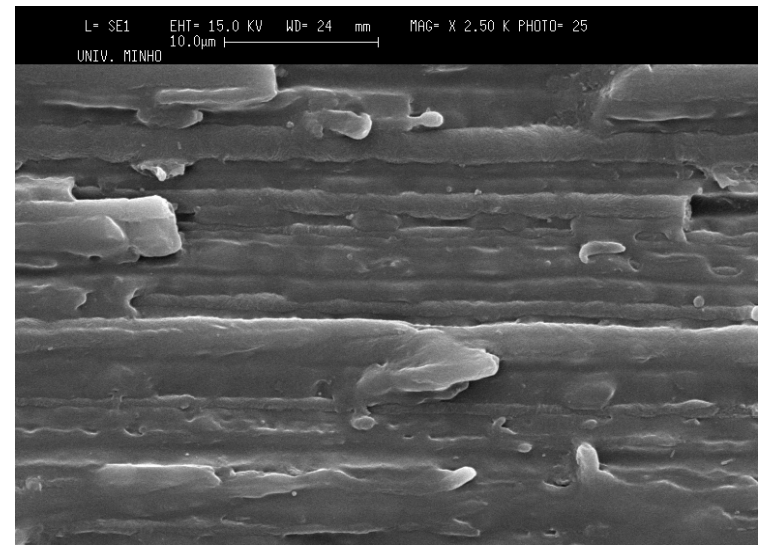

b)

Figure 3 - SEM micrographs of the samples a) with PLA and b) with PCL.

In the biodegradability tests of HDPE/PLA and HDPE/PCL blends were found to be readily colonized by a pure culture of Pseudomonas fluorescens in static culture growing conditions and to promote biofilm growth, as determined by comparing bacterial counts obtained after one and seven weeks of experiment lifetime (fig. 4). Interestingly HDPE was found not to be resistant to bacterial attachment as it did not support a net growth of the adhered cells.

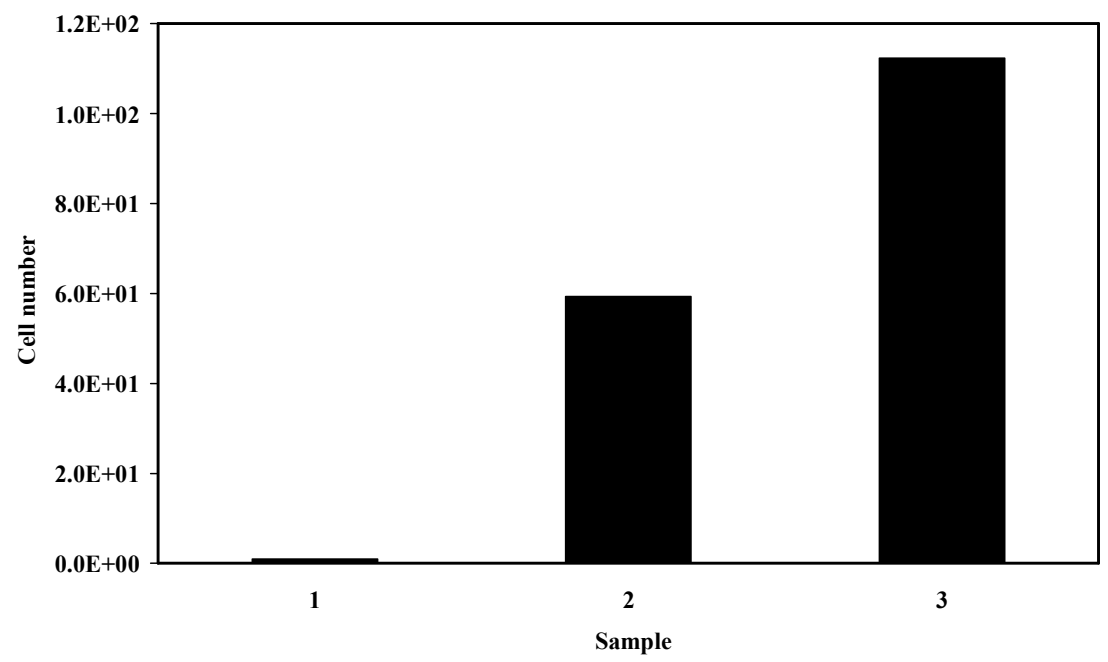

Figure 4 - Ratio between the biofilm bacteria number on materials tested quantified in the $7^{\text {th }}$ week and in the $1^{\text {st }}$ week of experiment lifetime.

All tested materials supported the growth of Pseudomonas fluorescens in suspension (fig. 5). One possible explanation for this result is that Pseudomonas fluorescens secreted exodepolymerases that cleaved the high molecular weight polymer chains. The oligomers and monomers formed could be transported through cell membranes and served as a source of carbon and energy for bacterial suspended growth. The numbers of bacteria quantified in suspension for all the tested materials were lower than the ones attached to the materials. This result might be explained by the fact that bacteria in the surface of the material have direct access to the organic carbon (shorter diffusion distance) and consequently grow faster. These experimental results suggest that HDPE/PCL from 
all the materials tested presents the highest degree of biodegradability, as determined by comparing bacterial counts.

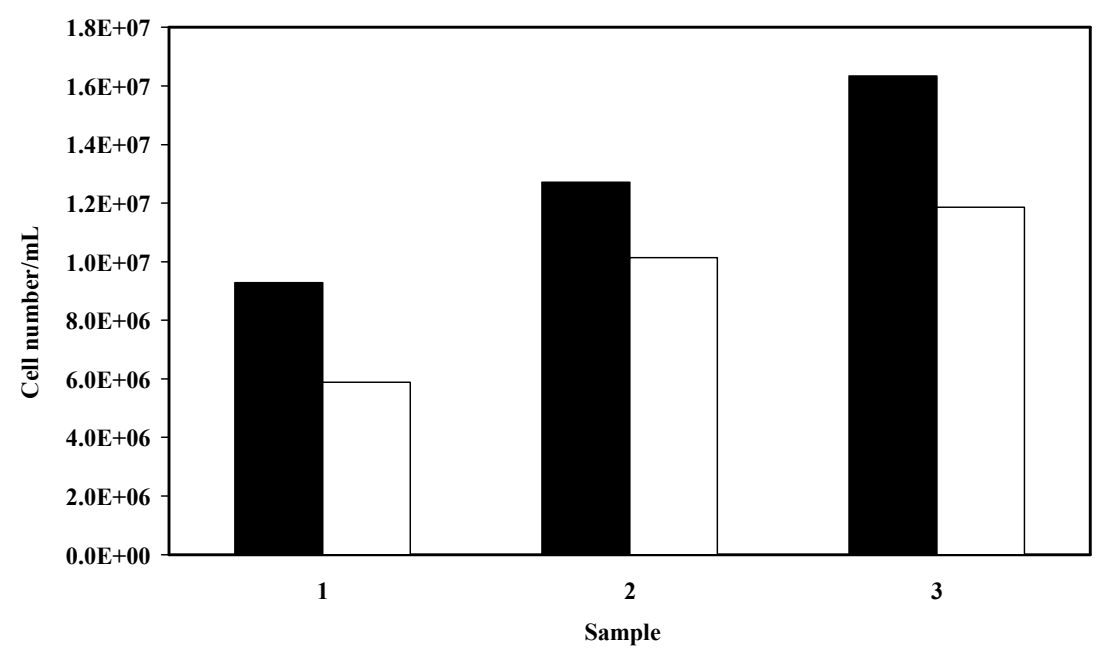

Figure 5 - Numbers of Pseudomonas fluorescens on materials tested (black bars) and in suspension (white bars) quantified in the $7^{\text {th }}$ week of experiment lifetime.

\section{Summary}

The purpose of this work was to prepare blends with a synthetic polymer (HDPE) and biodegradable polymers (polylactic acid and poly ( $\varepsilon$-caprolactone), in order to obtain biodegradable materials with distinct mechanical properties. They were prepared in a co-rotating twin-screw extruder together with a polyethylene modified with maleic anhydride used as compatibiliser.

The mechanical results showed that the addition of PLA leads to materials with a higher modulus and a lower elongation at break than HDPE. Conversely, the addition of PCL increases the elongation at break and weakens, as expected, the Young modulus. The biodegradability tests performed showed that HDPE/PCL material is more biodegradable than HDPE/PLA blends and both more than the synthetic HDPE. The results mentioned above suggest that blending synthetic and biodegradable polymers is an effective route to produce materials with a range of mechanical properties, keeping its biodegradability.

\section{References}

[1] L. Jiang, M. Wolcott and J. Zhang: Biomacromolecules Vol. 7 (2006), p. 199.

[2] M. S. Lindblad, Y. Liu, A. C. Albertsson, E. Ranucci and S. Karlsson: Adv. Polym. Sci. Vol. 17 (2002), p.139.

[3] S. Ray and M. Bousmina: Prog. Mat. Sci. Vol. 50 (2005), p. 962.

[4] S. Wang and J. Yu: J. Appl. Polym. Sci. Vol 93 (2004), p. 686.

[5] D. Bikiaris, J. Prinos, K. Koutsopoulos, N. Vouroutzis, E. Pavlidou, N. Frangi and C. Panayiotou: Polm. Degrad. Stab. Vol. 59 (1998), p. 287.

[6] G. Biresaw and C.J. Carriere: Composites- Part A Vol. 35 (2004), p. 313.

[7] A. Mathew, K. Oksman and M. Sain: J. Appl. Polym. Sci., Vol. 97 (2005), p. 2014. 